\title{
On the Role of Nerve Growth Factor in the Development of Myelinated Nociceptors
}

\author{
Gary R. Lewin, Amy M. Ritter, ${ }^{a}$ and Lorne M. Mendell \\ Department of Neurobiology and Behavior, State University of New York at Stony Brook, Stony Brook, New York 11794
}

\begin{abstract}
We have previously demonstrated that administration of antisera against NGF (anti-NGF) can have profound effects on developing primary afferents (Ritter et al., 1991). Chronic administration of anti-NGF to rats beginning on the day of birth results in a severe depletion of cutaneous A $\delta$ highthreshold mechanoreceptors (HTMRs) from the sural nerve. Here we have carried out further experiments in order to define the period of time over which this change in the cutaneous afferent population can be produced, and to investigate a possible mechanism for the change. Treatment with anti-NGF from postnatal day (PND) 0-14 resulted in a depletion of cutaneous A $\delta$ HTMAs from the sural nerve and also a $\mathbf{2 0} \%$ loss of sensory neurons. However, treatment from PND 2-14 produced an identical deficit of HTMRs without any accompanying cell death. Thus, the depletion of cutaneous A $\delta$ HTMRs can be achieved in the absence of cell death induced by anti-NGF treatment. It was also found that a 7 d treatment from PND 4-11 was sufficient to reproduce this effect, but that $7 \mathrm{~d}$ treatments earlier (PND 2-9) or later (PND 7-14) within the first 2 weeks were much less effective. This critical period, PND 4-11, corresponds to a period of anatomical change in the innervation of the skin, from epidermal innervation to primarily dermal innervation (Fitzgerald, 1967; Reynolds et al., 1991). In every case where antiNGF treatment reduced the proportion of HTMRs, there was a reciprocal increase in the proportion of sensitive $A \delta$ hair follicle (D-hair) afferents. We hypothesize that in the absence of NGF, developing cutaneous A $\delta$ HTMRs do not die but innervate novel targets in the dermis and become D-hair afferents instead.
\end{abstract}

The development of rat sensory neurons continues well after birth and is not completed until at least $14 \mathrm{~d}$ postnatally (PND 14). Responses in primary afferents can be elicited by stimulation of the skin as early as embryonic day 16 (E16) (Fitzgerald, 1987 a). On the day of birth, slowly adapting and rapidly adapting afferents can be distinguished, but responses are not mature

Received Sept. 17, 1991; revised Nov. 21, 1991; accepted Dec. 18, 1991

We thank Dr. Norbert Kremer for his help in preparing and characterizing the antisera, Dr. Simon Halegoua for the use of his facilities for this purpose, Kol Zarember for his technical assistance, and Dr. Sheryl Scott for her helpful comments on the manuscript. A.M.R. was supported in part by Training Grant MH

18018 (NIMH). This research was supported by a subproject of NIH Program Project Grant P01 NS 14899. Additional support was derived from NIH R01 NS 16966 to L.M.M. (Javits Neuroscience Award).

Correspondence should be addressed to Lorne $M$. Mendell at the above address

a Present address: Laboratory of Neural Control, NINDS/NIH, Building 36 , Bethesda, MD 20892.

Copyright (C) 1992 Society for Neuroscience $0270-6474 / 92 / 121896-10 \$ 05.00 / 0$ and do not resemble those of the adult until the second postnatal week (Fitzgerald, 1987b).

Somal spike shape also does not mature until PND 14. On PND 1, all cells have long-duration action potentials with an inflection on the falling phase. By PND 14, spikes in cells with unmyelinated axons (C-neurons) look much the same, but action potential duration has shortened a great deal in A-neurons, and most have lost the inflection (Fulton, 1987). This period between birth and $14 \mathrm{~d}$ also corresponds to the time during which peripheral myelination is completed (Friede and Samorajske, 1968).

NGF is necessary in utero for the development of most sensory neurons (Johnson et al., 1980). While not required for survival in the adult, around $50 \%$ of sensory neurons maintain high affinity receptors (Richardson et al., 1986) and continue to transport NGF retrogradely throughout adult life (Thoenen and Barde, 1980). It has been reported that administration of NGF antisera beginning on the day of birth leads to the death of between 18 and $38 \%$ of sensory neurons. The cells that are lost after this treatment are primarily the small $\mathrm{C}$ neurons (Yip et al., 1984; Hulsebosch et al., 1987). Exposure to antibodies against NGF in the adult produces no cell death (Gorin and Johnson, 1980; Schwartz et al., 1982), but it is not known when during development the dependence on NGF for survival ends.

We have demonstrated previously (Ritter et al., 1991) that chronic administration of NGF antisera beginning on the day of birth causes a change in the physiology of A $\delta$ cutaneous afferents, thinly myelinated afferents that in the young adult rat conduct between $2-14 \mathrm{~m} / \mathrm{sec}$. Normally, the majority of A $\delta$ afferents in the sural nerve innervate cutaneous high-threshold mechanoreceptors (HTMRs) and respond to noxious mechanical stimulation of the skin. After NGF deprivation beginning on the day of birth, most A $\delta$ afferents innervate D-hairs, lowthreshold mechanoreceptors that are exquisitely sensitive to hair movement (Burgess and Perl, 1967; Lynn and Carpenter, 1982). We have suggested that this change may not be due simply to selective death of developing HTMRs caused by anti-NGF treatment, but might involve redirection of fibers that would have become HTMRs to innervate D-hairs instead. Here we further define the period during which anti-NGF treatment can alter the composition of the sural nerve, and examine more closely the issue of whether cell death can be responsible for this change.

Portions of these data have been presented in short form (Lewin et al., 1991a; Mendell et al., 1991; Ritter et al., 1991).

\section{Materials and Methods}

Two antisera were raised in adult rabbits against mouse $2.5 \mathrm{~S}$ NGF and used interchangeably throughout these experiments. Sera were tested for their ability to block NGF-induced neurite outgrowth in PC12 cells 


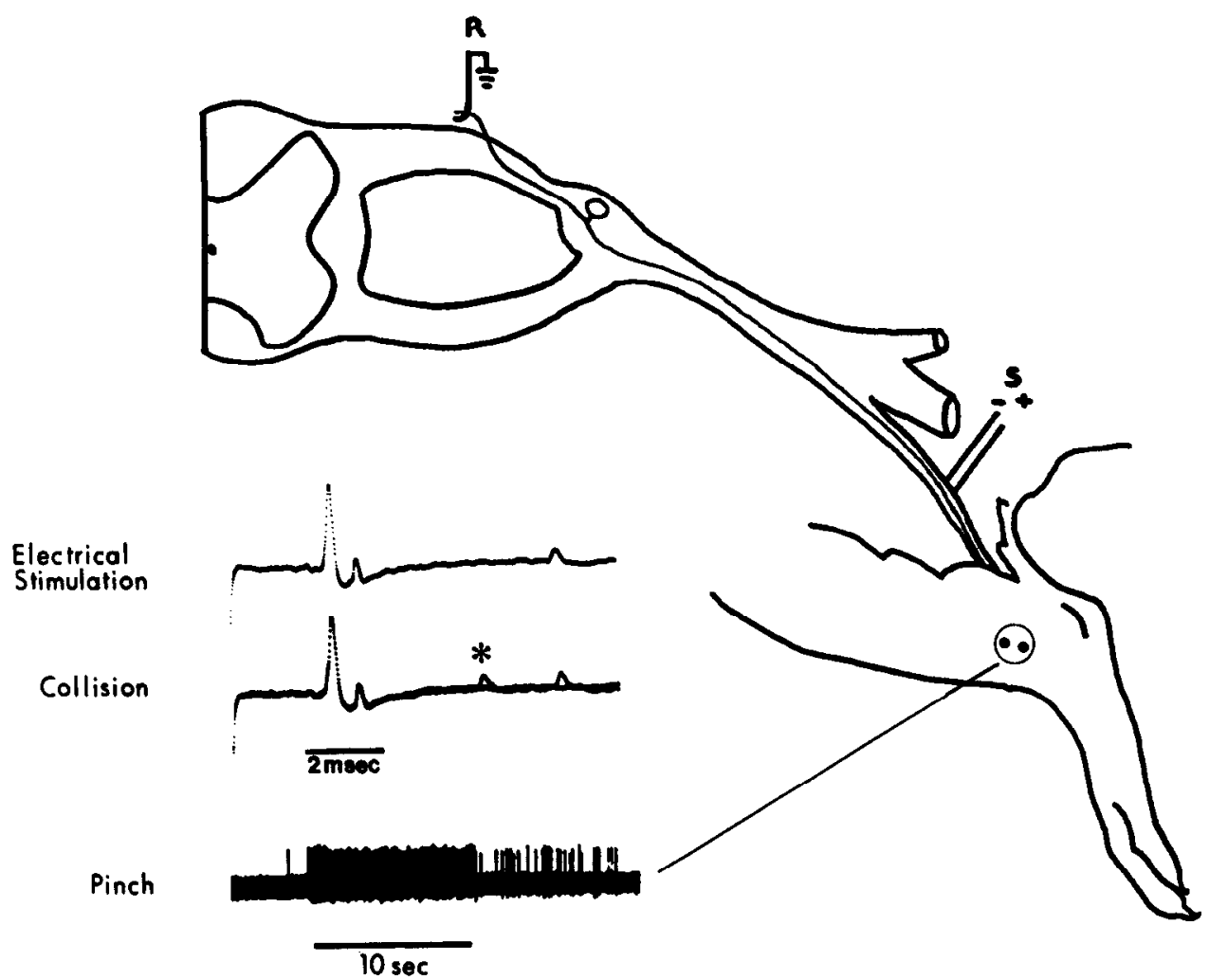

Figure 1. Extracellular recordings were made from teased dorsal root filaments of units driven by sural nerve stimulation. Three units were present in this strand that were easily distinguishable on the basis of the size and shape of their spikes (top record). The slowestconducting $(5.5 \mathrm{~m} / \mathrm{sec})$, and smallest, unit was identified as a cutaneous HTMR. The middle trace shows two superimposed traces before and after natural stimulation of the receptive field in the periphery (in this case, pinching of the receptive field). The nerve was stimulated at high frequencies $(5-10 \mathrm{~Hz})$ to increase the chance that a naturally evoked spike would abolish an electrically evoked spike due to refractoriness. The asterisk indicates the naturally evoked spike, which in this case abolished the spike evoked by nerve stimulation. The bottom trace shows the same unit in response to pinch of its receptive field. This was an example of a cutaneous HTMR with a slowly adapting discharge. The gain in the bottom record is twice that of the top two records.
(Greene, 1977a) and to recognize NGF on Western blots. At the end of the series of experiments, the bioassay was repeated, and both sera were still able to block the effects of $50 \mathrm{ng} / \mathrm{ml}$ of NGF at a concentration of $1 / 1000$.

Sprague-Dawley rats of either sex were injected for various times with antisera $(5 \mu \mathrm{l} / \mathrm{gm}$, s.c.) every day for the first $7 \mathrm{~d}$, and then every other day for the duration of the treatment. The day of birth was denoted PND 0, and treatments were given from PND 0-14, PND 2-14, PND 2-9, PND 4-11, or PND 7-14. Additionally, data are included from animals treated from birth to 5 weeks of age, animals treated from 0 to 5 weeks and allowed to survive untreated for an additional 3 weeks, and animals treated from 2 to 5 weeks of age (Ritter et al., 1991). Recordings were made from at least three animals in each treatment group, and control data were taken from untreated age-matched animals. Usually about 15 units were characterized in each animal so that the afferents recorded in each animal contributed equally to the total. It was noted that the proportions of afferent types in individual animals always approximated those found for the entire group (Ritter et al., 1991).

Electrophysiological recordings were made under urethane anesthesia $(1.25 \mathrm{gm} / \mathrm{kg})$ after the animals had reached $5-8$ weeks of age. A laminectomy was performed to expose the entire L5 dorsal root and recordings were made in teased dorsal root filaments of single units driven by sural nerve stimulation as described previously (Lewin and McMahon, 1991). Each single unit activated by electrical stimulation of the sural nerve was characterized as to adequate stimulus (Horch et al., 1977 ) with the aid of a dissecting microscope so that individual hairs could be manipulated. Conduction velocity, receptive field size and location, and von Frey threshold were also noted. For cutaneous HTMRs, von Frey thresholds were always determined from the most sensitive spot in the receptive field, and for slowly adapting HTMRs, threshold was considered to be the lowest value of force that would produce a slowly adapting discharge. In the rat, cutaneous HTMRs in hairy skin do not respond well to heat (Lynn and Shakhanbeh, 1988); therefore, responses to heat were not tested in these experiments. The ability of naturally evoked impulses to abolish electrically evoked impulses due to refractoriness was tested to confirm further that they were generated in the same afferent (Fig. 1).

$A \beta$ afferents (those with conduction velocities greater than $14 \mathrm{~m} / \mathrm{sec}$ ) were consistently sampled in only three treatment groups: control, $0-5$ weeks treatment, and treatment from 0 to 5 weeks with an additional
3 weeks without treatment. After it became apparent that there was no change in $\mathrm{A} \beta$ physiology (see Results), only $\mathrm{A} \delta$ afferents (conduction velocity between 2 and $14 \mathrm{~m} / \mathrm{sec}$ ) were characterized in subsequent experimental groups. However, in every experiment across all treatment groups, an attempt was made to characterize every unit with a latency appropriate for A $\delta$ conduction velocity. Since the criterion for selecting units was latency to electrical stimulation and not response to natural stimulation, we consider that our sample accurately reflects a cross section of the A $\delta$ population. A few A $\delta$ units were isolated for which no receptive field was found. These never represented more than $8 \%$ of the $A \delta$ population in any given treatment group including controls and were excluded from the analysis. Receptive fields were found for all $A \beta$ afferents.

At the end of an experiment, some animals were perfused through the heart with warm saline followed by $2 \%$ paraformaldehyde. L5 and L5 dorsal root ganglia were removed, postfixed overnight, and stored in $30 \%$ sucrose. Frozen $30 \mu \mathrm{m}$ sections were cut and stained with cresyl violet, and raw profile counts were made from the ganglia. In this particular strain of rats (Sprague-Dawley, Taconic Farms, Germantown, NY), only these two ganglia contributed to the sciatic nerve. In every animal, the contributions of L4 and L5 to the sciatic varied: usually, the $\mathbf{L} 4$ ganglion was larger than the L5 ganglion, but sometimes they were nearly equal in size. To minimize this particular source of variability, counts from the two ganglia were pooled and counted as a single unit. This strategy has been shown to minimize bilateral asymmetries in sensory neuron counts (Ygge et al., 1981). Approximately 10\% of the profiles randomly distributed through the two ganglia were drawn with the aid of a drawing tube, and mean profile diameters were measured on a digitizing tablet connected to the Eutectics neuron reconstruction system. A correction factor for the raw counts was calculated from the profile diameters using recursive translation (Rose and Rohrlich, 1987; McMahon et al., 1991). This is a mathematical correction factor for double counting that has certain advantages for the purposes of this study. The correction factor for any one ganglion is scaled according to the size distribution of cells present. Thus, a selective loss of small cells, for example, leads to an automatic compensatory change in the correction factor. The recursive translation program produces a reconstructed cell-size histogram based on the distribution of neuronal profiles present. This enabled us to demonstrate a selective loss of small cells after antiNGF treatment (see Fig. 3).

A total of $166 \mathrm{~A} \beta$ afferents and $351 \mathrm{~A} \delta$ afferents were recorded from 


\section{Control}

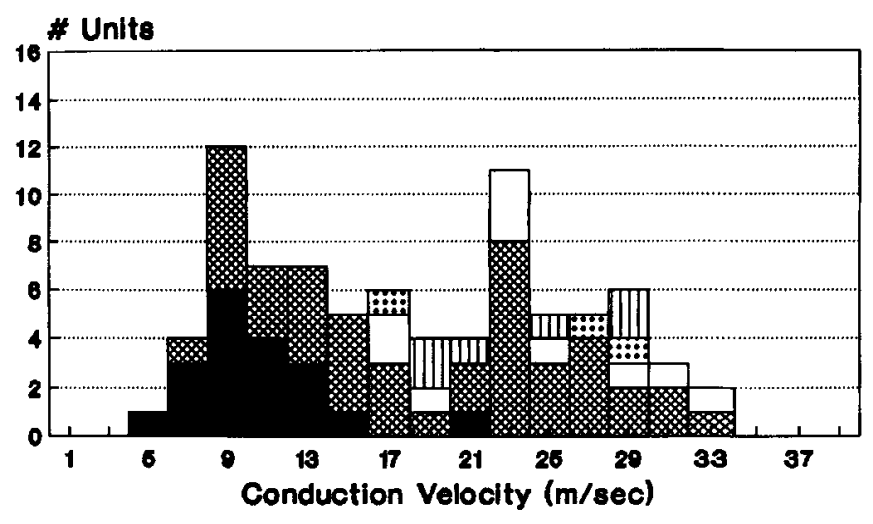

Anti-NGF (0-5 weeks)

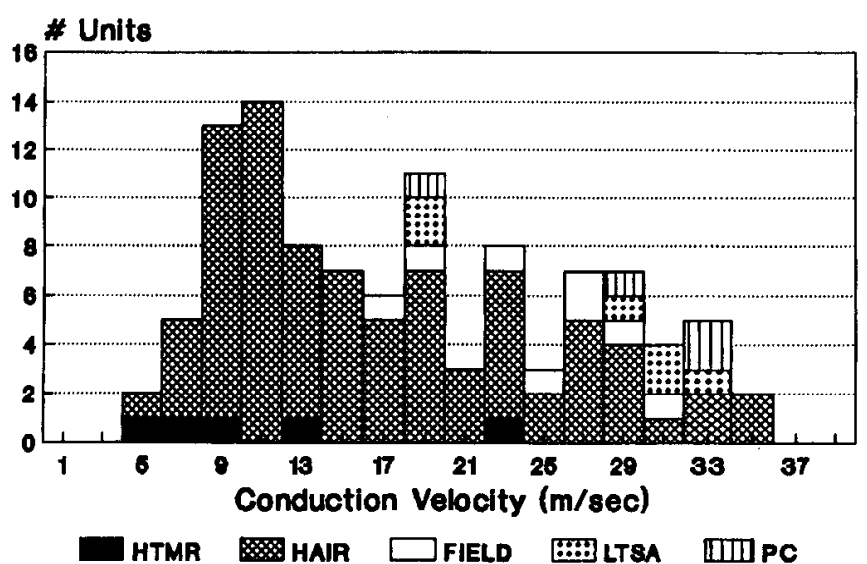

Figure 2. Plot of numbers of units broken down by receptor type versus conduction velocity. It will be noticed that HTMRs conducting in the A $\delta$ range are markedly depleted after anti-NGF treatment and appear to be replaced by hair follicle afferents $(H A I R)$, in this case D-hair afferents. Within the $\mathrm{A} \beta$ range most afferents were hair follicle afferents $(H A I R)$, and this remained the case after anti-NGF treatment. AntiNGF did not appear to affect the proportions or conduction velocities of the minority $\mathrm{A} \beta$ afferents such as Pacinian corpuscle afferents $(P C)$, low-threshold slowly adapting afferents (SAI and SAII) ( $L T S A)$ or field receptors $(F I E L D)$ found in the sural nerve (see Table 1 for more details). For these experiments, a total of 99 units were recorded in control animals and 105 units in anti-NGF-treated animals. and fully characterized in these experiments. All means quoted in the text are given together with their standard deviations.

\section{Results}

\section{Physiology of $A \beta$ afferents}

Table 1 illustrates the percentages of different types of cutaneous $\mathrm{A} \beta$ afferents in control animals and in those treated from birth to 5 weeks with anti-NGF. The latter group was an amalgamation of data from animals that were treated with antiserum up until the time of recording at 5 weeks of age $(n=5)$ and from animals that were treated for 5 weeks and allowed to survive without treatment for an additional 3 weeks before recording $(n=3)$. These data were pooled since no significant differences were seen between these two groups in the proportions or the physiology of $\mathrm{A} \beta$ or $\mathrm{A} \delta$ afferents. When the data from antiNGF-treated animals are compared to control data, it can be seen that the proportions of hair follicle afferents and other cutaneous afferents (field receptors, slowly adapting type $1, \mathrm{~Pa}$ cinian corpuscles, and A $\beta$ HTMRs) were very similar, differing by only $11 \%$ in the case of hair follicle afferents and by no morc than $6 \%$ for any of the other afferent types listed above $(p>$ $0.3, \chi^{2}$ ). Physiological parameters of cutaneous afferents (see Fig. 1) such as receptive field size, location, response to natural stimulation, and von Frey thresholds (Table 1) were indistinguishable from controls. Table 1 includes only cutaneous units; units with subcutaneous receptive fields, that is, "deep" units, accounted for about a third of the total $A \beta$ afferents sampled, $28 \%$ in control and $32 \%$ in anti-NGF-treated animals. These $\mathrm{A} \beta$ "deep" units were often low threshold and slowly adapting.

Mean conduction velocities for $A \beta$ afferents were remarkably similar in control and anti-NGF-treated animals (Table 1). This is further illustrated in Figure 2, which shows the conduction velocity of all cutaneous units broken down by afferent type. Control and anti-NGF histograms differ only in the virtually complete depletion of A $\delta$ cutaneous HTMRs from the treated groups; $A \beta$ afferents are represented in similar proportions and at similar conduction velocities in both groups. This is in agreement with the finding that chronic anti-NGF treatment does not change the distribution of myelinated axon diameters in the sural nerve (Ritter et al., 1991).

\section{Lack of correlation with cell death}

We have previously demonstrated that treatment of rats with antisera beginning at 2 weeks of age does not produce the change in physiology seen after treatment beginning on the day of birth (Ritter et al., 1991; see also Fig. 5). This implies that it should be possible to create a deficit of cutaneous HTMRs by treatment only in the first two postnatal weeks. Indeed, treatment with

Table 1. Physiology of $A \beta$ afferents

\begin{tabular}{|c|c|c|c|c|c|c|}
\hline & \multicolumn{3}{|l|}{ Control } & \multicolumn{3}{|l|}{ Anti-NGF } \\
\hline & $\%$ Total & $\begin{array}{l}\text { Mean CV } \\
(\mathrm{m} / \mathrm{sec})\end{array}$ & $\begin{array}{l}\text { von Frey } \\
\text { threshold (g) }\end{array}$ & \% Total & $\begin{array}{l}\text { Mean CV } \\
(\mathrm{m} / \mathrm{sec})\end{array}$ & $\begin{array}{l}\text { von Frey } \\
\text { threshold (g) }\end{array}$ \\
\hline Hair follicle afferent & $58.8 \%(30 / 51)$ & $22.9 \pm 5.3$ & $0.05 \perp 0.08$ & $69.3 \%(43 / 62)$ & $22.5 \pm 6.0$ & $0.05 \pm 0.08$ \\
\hline Slowly adapting (SAI and SAII) & $5.9 \%(3 / 51)$ & $24.2 \pm 5.1$ & $0.07 \pm 0.0$ & $9.7 \%(6 / 62)$ & $26.6 \pm 6.0$ & $0.05 \pm 0.02$ \\
\hline Field & $17.6 \%(9 / 51)$ & $24.0 \pm 5.8$ & $0.19 \pm 0.2$ & $12.9 \%(8 / 62)$ & $24.4 \pm 4.6$ & $0.38 \pm 0.27$ \\
\hline Pacinian corpuscle & $12 \% \quad(6 / 51)$ & $23.8 \pm 4.0$ & NA & $6.5 \%(4 / 62)$ & $28.7 \pm 6.2$ & NA \\
\hline $\mathrm{A} \beta$ HTMR & $5.9 \%(3 / 51)$ & $20.1 \pm 4.3$ & $11.88(n=1)$ & $1.6 \%(1 / 62)$ & 23.4 & 11.8 \\
\hline
\end{tabular}


A
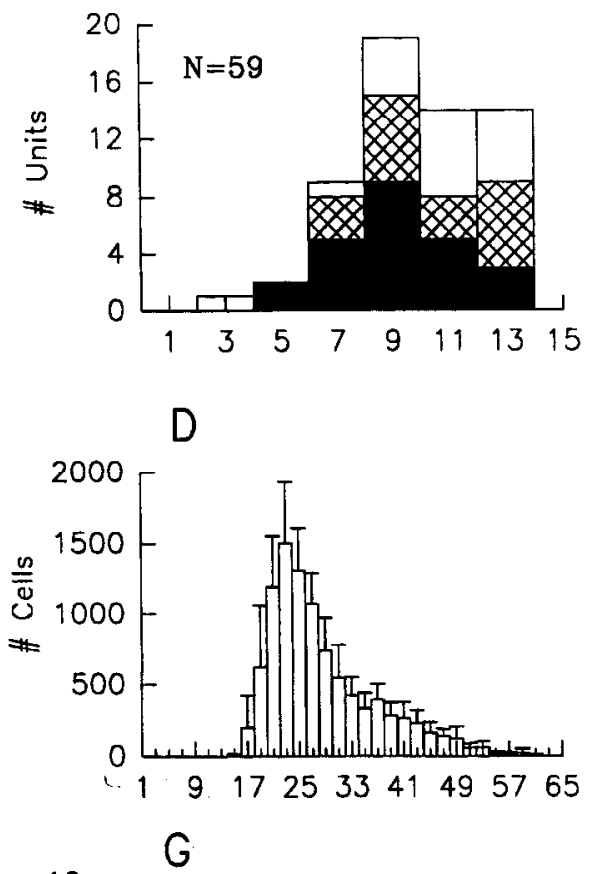

$G$
B

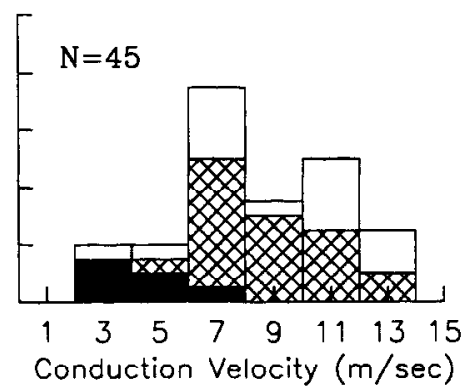

E

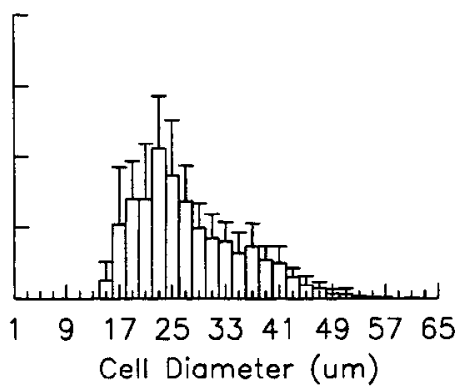

C

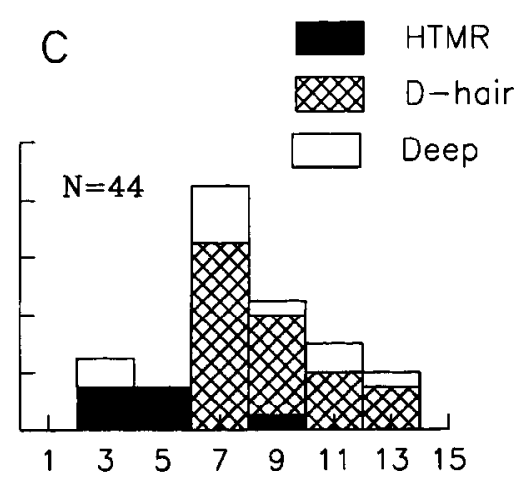

$\mathrm{F}$

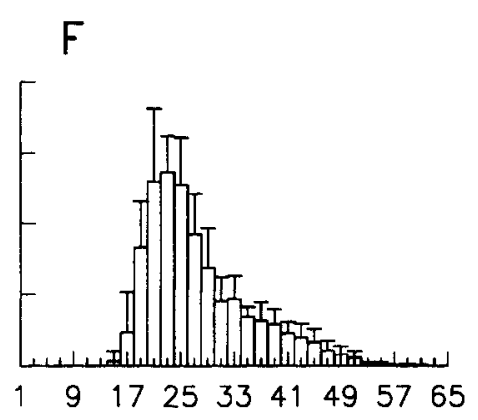

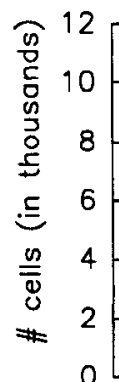

Figure 3. $A-C$ are distributions of receptor type versus conduction velocity of A $\delta$ afferents from control and PND 0-14 and PND 2-14 antifrom the population in control animals $\left(p<0.01, \chi^{2}\right)$. For control, $n=9$ animals, and for PND $0-14$ and PND $2-14, n=3$ each. $D-F$ are cellsize histograms generated by recursive translation for control and PND 0-14 and PND 2-14 treated groups, respectively. $G$ is a graph of mean cell counts pooled from L4 and L5 dorsal root ganglia from animals treated as indicated. Counts were made from ganglia from both sides of each animal. For control, $n=5$ animals, for PND $0-14, n=6$ animals, and for PND 2-14, $n=5$ animals. Note that anti-NGF treatment from PND 0 to 14 and 2 to 14 produces essentially the same depletion of HTMRs from the sural nerve $(A-C)$. Treatment beginning at birth leads to a significant loss of sensory neurons from the L4 and L5 ganglia $(G)$, which appeared to be specific for small neurons. In contrast, anti-NGF treatment from PND 2 to 14 produced no significant cell death $(F$ and $G)$ even though HTMRs were similarly depleted from the nerve $(C)$.

antisera from PND 0 to PND 14 caused a loss of cutaneous HTMRs similar to, although not as extensive as, that seen in chronically treated animals (0-5 weeks; see Fig. 2). In control animals, cutaneous HTMRs represent the largest proportion of the A $\delta$ population, constituting $41 \%$ of the total sample. D-hairs make up $29 \%$ of the sample, and the remaining $30 \%$ are "deep" units that innervate subcutaneous tissue (Fig. $3 A$ ). After antiNGF treatment from 0 to $14 \mathrm{~d}$, cutaneous HTMRs represented only $13 \%$ of the total $\mathrm{A} \delta$ population. The proportion of $\mathrm{D}$-hairs had increased to $51 \%$, while deep units remained little changed at $36 \%$ (Fig. $3 B$ ).

It was found that anti-NGF treatment beginning on the day of birth resulted in a significant decrease in the number of DRG cells. This loss of $20 \%(p<0.05$, unpaired $t$ test) (Fig. $3 G)$ is similar to results obtained by others (Yip et al., 1984). It is evident from the large SE bars in Figure $3 G$ that these cell counts were highly variable. This variability arose from two sources. First, counts from animal to animal, even in controls, were variable. Second, the cell death induced by anti-NGF did not occur in all cases. In some cases, cell counts from ganglia from one side of a treated animal were close to the mean for control animals, but on the contralateral side the number of cells could be reduced by half. In addition, in one animal the number of cells present after anti-NGF was essentially the same as found in controls. This suggested to us that these cells may not be sensitive to NGF deprivation for long after birth, and that perhaps small differences in the ages of animals at the onset of antiNGF administration can lead to large differences in the amount of anti-NGF induced cell death.

The cell size histograms (Fig. $3 D-F$ ) from each treatment group exhibit a clear bimodal distribution, with a large peak presumably corresponding to the small sized C-neurons and a 


\section{CONTROL}

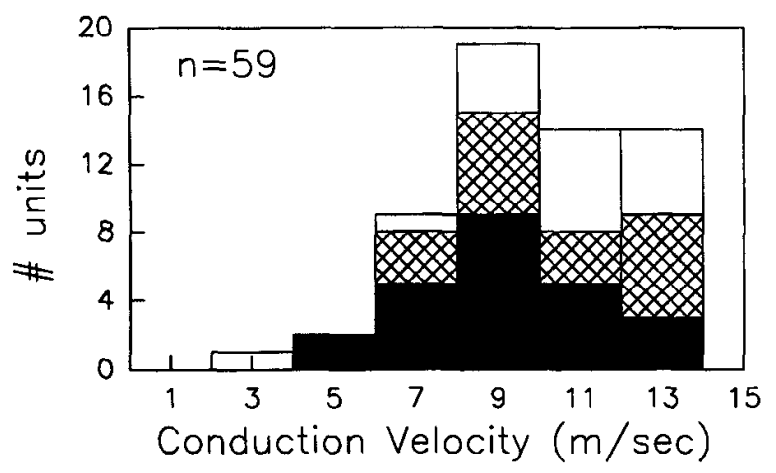

ANTI-NGF PND 4-11

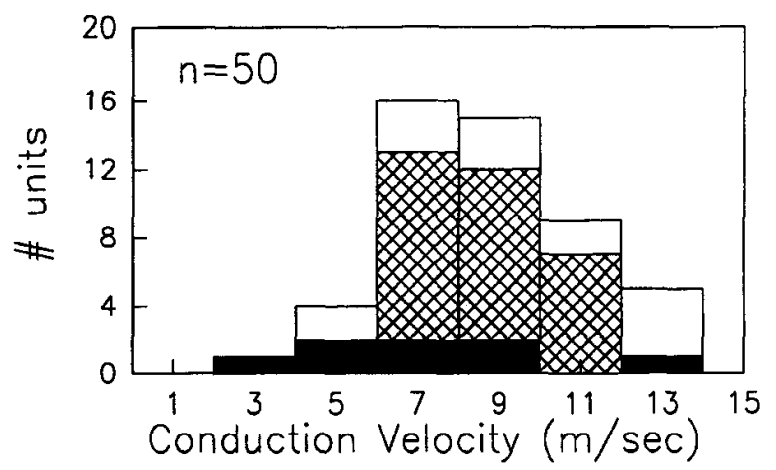

ANTI-NGF PND 2-9

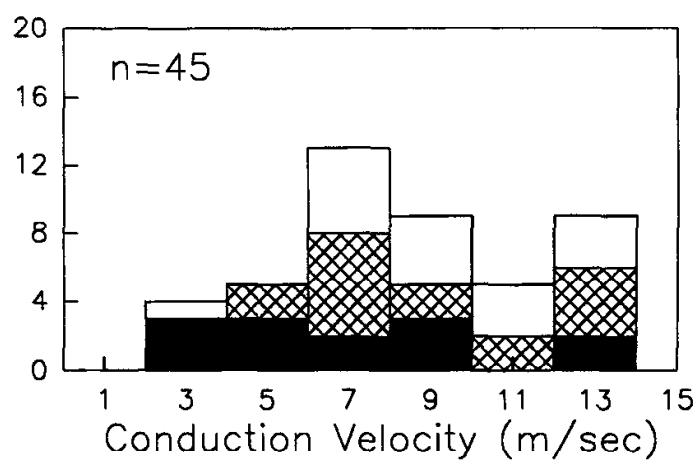

ANTI-NGF PND 7-14

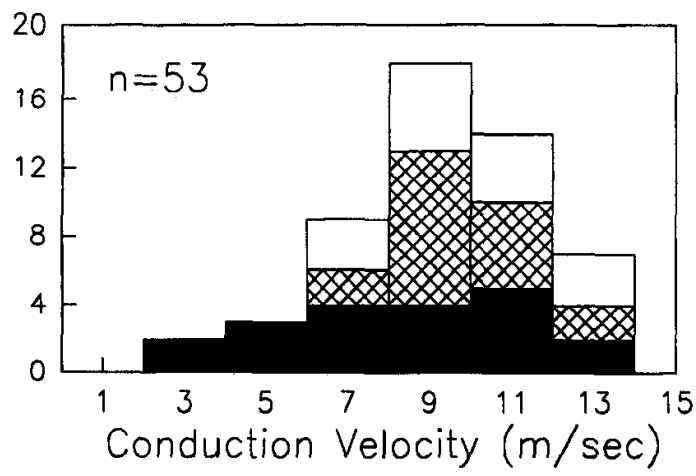

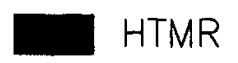

D-hair

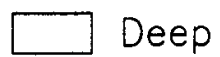

Figure 4. Histograms of receptor type of A $\delta$ afferents versus conduction velocity for control and anti-NGF-treated animals. For control, $n=9$, and for all other groups, $n=3$. Cutaneous HTMRs are significantly reduced in animals treated from PND 4 to $11\left(p<0.01, \chi^{2}\right)$ but not in animals treated from PND 2 to 9 or PND 7 to $14\left(p>0.2, x^{2}\right)$. Thus, a $7 \mathrm{~d}$ anti-NGF treatment within the first 2 postnatal weeks is only fully effective when given between PND 4 and 11 and not when given earlier (PND 2-9) or later (PND 7-14) in development.

smaller peak corresponding to the larger A-neurons (Lawson, 1979; Lawson et al., 1984). The cell loss from the 0-14 d treated animals seems to be restricted to the first peak and probably represents a loss of C-neurons (Harper and Lawson, 1985; Fig. $3 E)$.

Anti-NGF treatment beginning later, on PND 2-14, produced a deficit in cutaneous HTMRs almost identical to that seen in animals treated from PND 0 to 14. Cutaneous HTMRs represented only $16 \%$ of the sural nerve A $\delta$ population in these animals (Fig. 3C). However, this treatment produced no discernible cell death since cell counts from PND 2-14 d treated animals did not differ from those in control animals $(p>0.5$, unpaired $t$ test) (Fig. 3G). The fact that identical physiology is obtaincd with or without a $20 \%$ loss of cells leads us to conclude that the changes in physiology seen here are not correlated with cell death produced by anti-NGF treatment.

\section{Critical period}

Treatments were given for discrete $7 \mathrm{~d}$ periods during the first two postnatal weeks in an effort to determine more precisely when this change could be produced, that is, a developmental critical period. Figure 4 illustrates that treatment late in the postnatal period, from PND 7 to 14 , was ineffective at producing a deficit in cutaneous HTMRs. The proportions of units in these animals (38\% HTMRs, 34\% D-hairs, and 28\% "deep" units) were similar to the proportions in control animals. Likewise, treatment early in the postnatal period, from PND 2 to 9, did not significantly $\left(p>0.2, x^{2}\right)$ change the proportions of $\mathrm{A} \delta$ afferents, although there was a somewhat larger (but statistically insignificant) drop in the proportion of cutaneous HTMRs, down to $29 \%$. Surprisingly, treatment from PND 4 to 11 was sufficient to reduce the proportion of cutaneous HTMRs to $16 \%$, which is very similar to the deficit produced in animals treated from PND 0 to 14 and PND 2 to 14.

A summary of all the treatment groups is illustrated in Figure 5. From this figure, it is evident that across all treatment groups, the proportion of "deep" units remains constant, between $28 \%$ and $36 \%$. If cutaneous HTMRs were simply disappearing, or slowing their conduction velocity to become C-fibers (and therefore not being sampled), one might expect the proportions of "deep" units and D-hair afferents to rise equally. Instead, in every instance where there was a drop in the proportion of cutaneous HTMRs, there was a corresponding rise in the proportion of D-hair afferents, with no change in the proportion of 


\section{PERCENTAGES OF AFFERENT TYPES AFTER ANTI-NGF TREATMENTS}

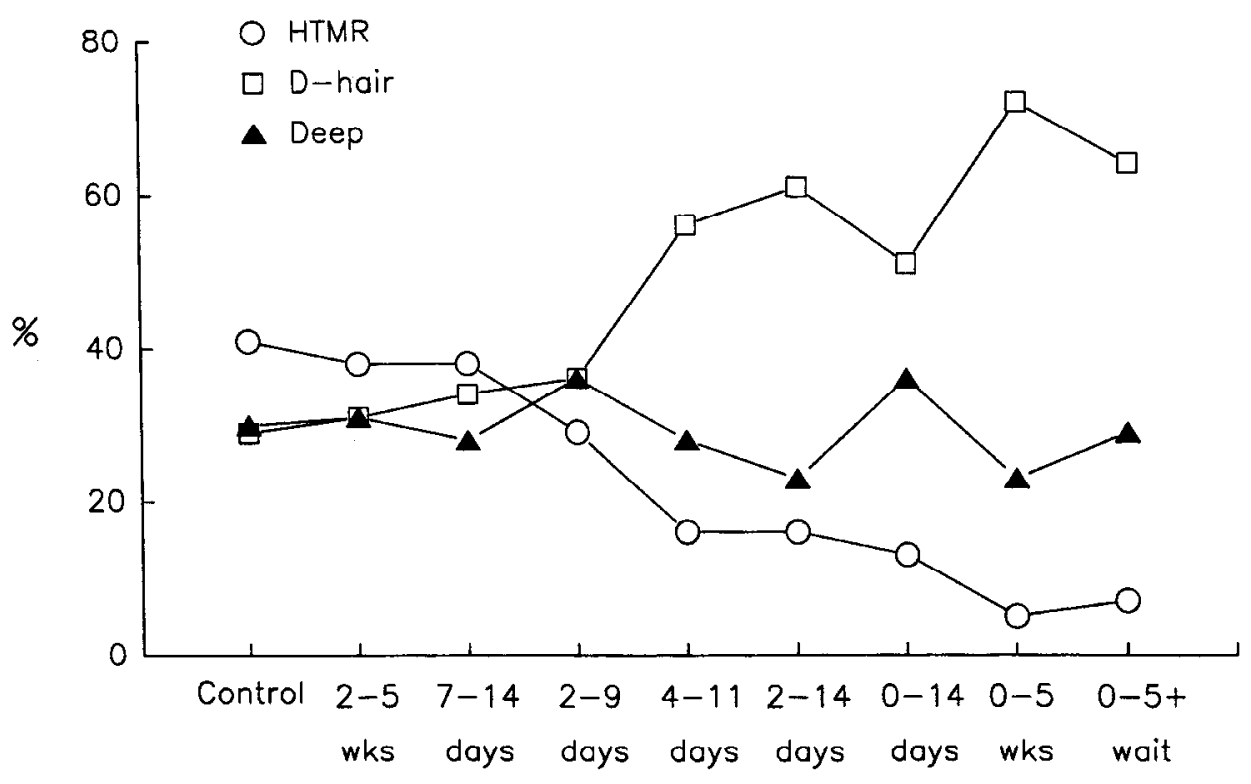

Treatment Duration
Figure 5. Percentages of A $\delta$ afferent types after various anti-NGF treatment regimes. Percentages for the 2-5 week, $0-5$ week, and $0-5$ week plus 3 week wait groups were reported previously (Ritter et al., 1991). Note that in each case where there was a large decrease in the percentage of HTMRs in the nerve, there was a reciprocal increase in the percentage of D-hair afferents. This happened in the absence of any consistent effect on the proportion of "deep" afferents.

\begin{abstract}
“deep" units. Thus, it appears that D-hair afferents replace the missing HTMRs.
\end{abstract}

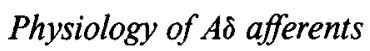

The physiology of D-hair afferents and "deep" units recorded in anti-NGF-treated animals did not differ from that in control animals. D-hairs in normal animals were extremely sensitive, having von Frey thresholds that averaged only $0.01 \pm 0.02 \mathrm{gm}$. These units were activated by seemingly all of the hairs within the receptive field, gave a brisk response to hair movement, and often were sensitive enough to be activated by small movements of the skin at sites distant from the receptive field. D-hair afferents in all of the anti-NGF-treated groups (both effective treatments and ineffective treatments) were normal, having von Frey thresholds averaging $0.01 \pm 0.02 \mathrm{gm}$ and responding identically to natural stimulation as D-hairs in controls. Neither the sizes nor the spatial distributions of the receptive fields on the limb in the anti-NGF-treated animals differed from those in control animals.

Units with subcutaneous receptive fields, "deep" units, in control animals were usually high threshold, requiring frankly noxious stimulation of deep tissues or joints to evoke a response. These units could be either slowly or rapidly adapting, and the rapidly adapting units tended to have particularly high thresholds. Care was taken to distinguish "deep" units requiring manipulation of the underlying tissue to produce a response. After anti-NGF treatment, "deep" units were qualitatively the same as in controls and still were largely high threshold.

A $\delta$ cutaneous HTMRs have been described in detail in the cat (Burgess and Perl, 1967) and in the rat (Lynn and Carpenter, 1982), and the physiology of these units observed in our experiments was very similar. In control animals, the majority of cutaneous HTMRs (79\%) were slowly adapting and the recep- tive fields of these contained a number of sensitive spots. Rapidly adapting units represented the remaining $21 \%$ of the HTMR population and were extremely insensitive, having mean von Frey thresholds of $54 \pm 49 \mathrm{gm}$, compared to $4.3 \pm 2.3 \mathrm{gm}$ for the slowly adapting units. Rapidly adapting units often desensitized rapidly or gave such a sluggish response to natural stimulation (one or two spikes to intense pinching of the receptive field) that determination of von Frey thresholds was often problematic.

In order to compare the physiology of cutaneous HTMRs after anti-NGF treatment, animals were subdivided into two categories, those in which anti-NGF treatment produced a loss of cutaneous HTMRs (designated "effective" in Fig. 6) and those in which anti-NGF treatments were without effect (designated "ineffective" in Fig. 6). Anti-NGF treatment that did not result in a deficit in HTMRs did not change the physiology of the HTMRs. The percentages of slowly and rapidly adapting units were $76 \%$ and $24 \%$, respectively, the same as in controls. The von Frey thresholds for both the rapidly adapting units (45 \pm $50 \mathrm{gm})$ and the slowly adapting units $(6.0 \pm 4.8 \mathrm{gm})$ were unchanged. The cumulative sum distribution of thresholds for slowly adapting units is illustrated in Figure 6, and this distribution is identical to that of controls.

In contrast, anti-NGF treatments that caused a reduction in the proportion of HTMRs resulted in a change in the physiology of the remaining HTMRs. The proportion of HTMRs that were slowly adapting increased to $91 \%$. Only 2 rapidly adapting units were found out of a total of 23 , and both of these were found in animals treated from PND 4 to 11 , the briefest effective treatment. The mechanical thresholds of the slowly adapting units were greater than in controls, averaging $9.5 \pm 6.7 \mathrm{gm}$ (Fig. 6). The cumulative sum distribution for these units was shifted to the right of both control and ineffective anti-NGF treated 


\section{HTMRs after Anti-NGF (SA units)}

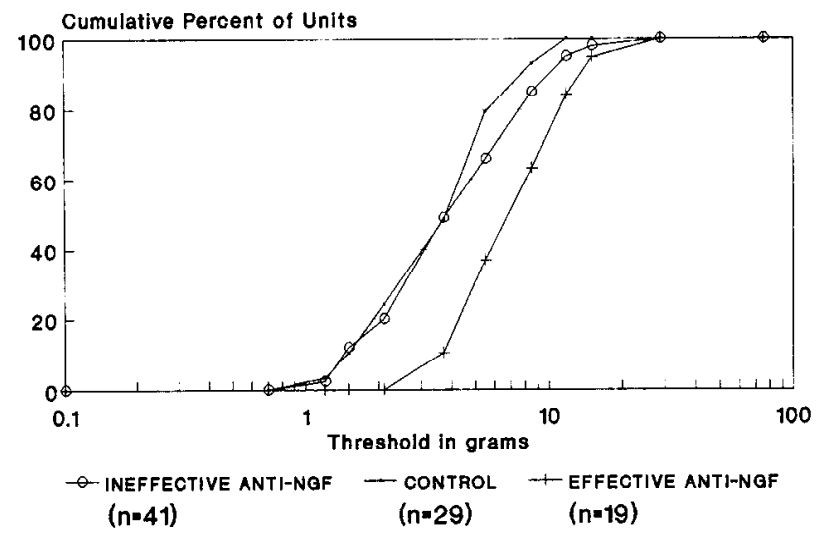

Figure 6. Cumulative sum distributions of mechanical thresholds of slowly adapting HTMRs from control and anti-NGF-treated animals measured with calibrated von Frey hairs. "Ineffective" treatments refer to those anti-NGF treatments that did not significantly reduce the number of IITMRs, and include PND 2-9, PND 7-14, and 2-5 week treatments. "Effective" treatments include PND 4-1 1, PND 0-14, PND 2$14,0-5$ week', and $0-5$ week plus wait groups. Mechanical thresholds are significantly elevated in cutaneous HTMRs from "effective" treatment groups $(p<0.001$, Kolmogorov-Smirnov) but not in cutaneous HTMRs from "ineffective" treatment groups $(p>0.3$, KolmogorovSmirnov).

animals. Thus, not only was there a large decrease in the number of HTMRs after anti-NGF treatment, but the few that remained were less sensitive as well.

\section{Discussion}

We have demonstrated that NGF deprivation during a brief critical period results in a deficit in cutaneous HTMRs and an increase in the mechanical thresholds of those HTMRs that remain. Concomitantly, there is an increase in the number of sensitive D-hair afferents. Since this change in the population of cutaneous afferents can occur in the absence of anti-NGFinduced cell death, we interpret it as switching of developing afferents from HTMRs into D-hairs.

The strength of the interpretation of this change as a phenotypic switch rather than a change caused by cell death rests on the fact that the phenomenology remains the same whether there is a large amount of cell death (0-14 d treatment) or no detectablc cell death (2-14 d treatment). However, we concede that if cutaneous HTMRs made up only $5 \%$ of the cells in the ganglia, and if only all of those died in the 2-14 d treatment, the large variability in these cell counts would make it possible to miss such a loss. If this were the case, it would imply these particular $\mathrm{A} \delta$ neurons are more susceptible to NGF deprivation than the smaller C-neurons; this seems unlikely. The finding that the percentage of D-hairs increases as the percentage of HTMRs decreases suggests that HTMRs do not die, but become D-hairs, since, if only HTMRs died, one would expect to sample roughly equal numbers of D-hairs and "deep" units as is the case in controls. Instead, one finds D-hairs and "deep" units at a ratio of roughly $2: 1$. This might be explained if some "deep" units die in addition to HTMRs, and the two types of cells are lost at some fixed ratio such that the proportion of "deep" units is decreased relative to D-hairs. However, such a mechanism would require a level of death of $A \delta$ neurons large enough to be detectable in cell counts. Perhaps the most compelling argument against cell death as a mechanism is the fact that anti-NGF treatment from PND 4 to 11 produces a more profound less of cutaneous HTMRs than a treatment of the same duration beginning earlier, from PND 2 to 9. Since the dependence of sensory neurons on NGF decreases with developmental age (Greene, 1977b), this result is inconsistent with the idea that the decrease in HTMRs is due to cell death.

It is possible that anti-NGF leads to the observed loss of HTMRs in the absence of cell death by specifically interfering with their development, by preventing either their myelination or the development of their receptor endings. Both these possibilities are operationally similar to cell death in that one would expect to see a selective loss of A $\delta$ fibers from the sural nerve and increased numbers of both deep and D-hair afferents (see above). These effects were never observed (Ritter et al., 1991). Furthermore, there was no increase in the proportion of units with no receptive field in anti-NGF-treated animals as might have been expected if receptor development had been prevented. In a recent study of C-fiber afferents after anti-NGF treatment (PND 2-14), no units were found with rcceptive ficlds resembling A $\delta$ HTMRs (G. R. Lewin and L. M. Mendell, unpublished observations). Thus, we feel that it is very unlikely that anti-NGF treatments deplete A $\delta$ HTMRs by interfering with myelin or receptor formation in the first 2 postnatal weeks.

The change in the composition of the A $\delta$ primary afferent population can be evoked by a relatively brief treatment over a critical period within the first two postnatal weeks. However, the deficit in cutaneous HTMRs observed after a brief neonatal treatment is not as great as that observed after chronic antiNGF treatment from 0 to 5 weeks. Several explanations can be offered for this discrepancy. The HTMRs that remain in the briefly treated neonatal group are among the slowest conducting afferents sampled (around half have conduction velocities less than $4 \mathrm{~m} / \mathrm{sec}$ ). Therefore, the larger numbers of HTMRs seen in animals subjected to brief treatment $(15 \%$ of the total compared to $5 \%$ in the chronically treated animals) might simply be due to sampling larger numbers of slow units in these treatment groups. Alternatively, there could be some small recovery in the neonatally treated animals: treatment was ceased on PND 14 , but recordings were not carried out until the animals had reached maturity, sometimes 4 or 5 weeks later. A third explanation would be that treatment is required beyond PND 14 to produce a maximal effect.

The additional finding that anti-NGF treatment can raise the mechanical threshold of cutaneous HTMRs under some circumstances is complicated by the fact that this occurs only in cases where the proportion of HTMRs is drastically reduced. Therefore, from these data it is not clear whether NGF has a direct effect on mechanical threshold, or whether the HTMRs that are "spared" represent a population that have higher thresholds than the population at large. However, in preliminary experiments we have found that treatment with exogenous NGF during the first 2 weeks can lower the mechanical threshold of cutaneous HTMRs (Lewin et al., 1991b). This would suggest a direct role for NGF in regulating mechanical threshold, but only during restricted periods in development.

The dosage regime we have chosen here produced a degree of sensory neuron cell death very similar to that of the regime used by Yip et al. (1984). This is despite the fact that they injected five times as much antisera during PND 0-7. Thus, we 
believe that the dosages we have used in these experiments are probably supramaximal for the effects we have seen, although we have no direct evidence that this is the case.

We believe the effects demonstrated here to be due specifically to a reduction in levels of NGF rather than in levels of any of the other members of the neurotrophin family. We have not explicitly tested our antisera for cross-reactivity with any of thesc othcr factors, but the known pattern of expression of $\mathrm{NGF}$ compared with that of NT-3 and brain-derived neurotrophic factor (BDNF) makes it likely that the effect seen here is mediated by NGF rather than by one of the related neurotrophic molecules. NGF and BDNF have been shown to support partially overlapping populations of sensory neurons in vitro (Lindsay et al., 1985), but levels of BDNF mRNA are low to undetectable in skin (Maisonpierre et al., 1990a,b). This would seem to rule out a crucial role for BDNF in the development of cutaneous innervation. NT-3 mRNA exists in high concentrations in skin but also is found in high concentrations in skeletal muscle and a variety of other non-neuronal tissues (Hohn et al., 1990; Maisonpierre et al., 1990a,b). Given this distribution, it would be difficult to explain how a factor affecting neurons innervating cutaneous but not those supplying subcutaneous structures could be NT-3. In contrast, levels of NGF and its message are high in skin and low in skeletal muscle (Korsching and Thoenen, 1983; Goedert et al., 1986; Davies et al., 1987), making it the most appropriately localized neurotrophic factor to mediate the stabilization of HTMRs described here.

A possible mechanism by which developing HTMRs might be induced to innervate hair follicles when NGF levels are lowered by antisera treatment is suggested by the sequence of events that occurs as sensory axons innervate skin. In the rat, scnsory axons grow out to the skin beginning around E13-E15. Axons reach trunk skin by $\mathrm{E} 13$ but do not reach the most distal skin in the toes until as late as E19 (Reynolds et al., 1991). Sensory axons do not immediately innervate targets in the skin but form a dense plexus in the epidermis that persists for the next several days (Fitzgerald, 1967; Reynolds et al., 1991). Most of these fibers then withdraw from the epidermis between PND 5 and 10 to innervate structures such as hair follicles in the dermis, with withdrawal of more rostral innervation occurring in advance of that of more caudal innervation. Some fibers remain in the epidermis in adulthood, and a proportion of these are believed to be the anatomical substrates of $A \delta$ cutaneous HTMRs (Kruger et al., 1981). Interestingly, it has been found that levels of NGF mRNA are more concentrated in the epidermis than in the dermis, at least in the embryo (Davies et al., 1987). We suggest that a relatively high level of NGF in the epidermis is necessary to stabilize the projection of developing A $\delta$ HTMRs, and that in its absence these cells do not die but withdraw to the dermis to innervate hair follicles.

A schematic diagram of the timing of skin innervation and the withdrawal of fibers from the epidermis is illustrated in Figure 7, and with it are shown the periods of time over which anti-NGF treatments were given. Only those injection periods that completely overlap the period of fiber withdrawal (PND 5-10) are effective in significantly reducing the numbers of cutaneous HTMRs. Anti-NGF treatment from PND 2 to 9 covers almost all the putative critical period and may produce a small loss of A $\delta$ HTMRs. However, NGF deprivation limited to the period between PND 4 and 11 is sufficient to produce a depletion of HTMRs equal to that found with anti-NGF treatments from PND 0 to 14 and 2 to 14 . Thus, it appears that NGF deprivation over the whole period of fiber withdrawal is necessary to produce the full effect.

If the phenomenon demonstrated here does represent a phenotypic switch, it would not be the first such demonstration in a neural crest derivative. Developing sympathetic neurons express noradrenergic markers when they initially innervate sweat glands, but are induced to switch to a cholinergic phenotype by a factor or factors present in the target tissue (reviewed in Landis, 1990). Here, the role for NGF in promoting the switch from HTMR to D-hair is not an instructive one. The presence of NGF would serve to provide a hospitable environment for developing HTMRs, with the actual switch being brought about by factors present in the dermis or produced by hair follicles. That this may represent a real switch in phenotype rather than just an exchange of targets is suggested by the following. Somal spikes recorded intracellularly from dorsal root ganglion cells innervating D-hairs in anti-NGF $0-5$ week treated animals all displayed the short-duration spike with a simple falling phase characteristic of these cells in normal animals. None was seen with the larger-amplitude, longer-duration spike with a biphasic falling phase that is characteristic of HTMRs (Ritter, 1991). Thus, at least this aspect of cell phenotype is in register with the target innervated.

The absence of cell death, the high correlation between the "critical period" for this effect and the timing of withdrawal of fibers from the epidermis, and the increase in the proportions of D-hairs all strongly support the notion of a change in developmental fate of HTMRs induced by NGF deprivation. Although the coincidence in timing between the physiological change and the anatomical change is striking, we have no direct evidence that the two are connected. Clearly, anatomical evidence will be needed to add further support for this hypothesis. Experiments are in progress to examine peptide staining in the skin and the ganglia after anti-NGF treatments. Indeed, preliminary studies show fibers immunoreactive for calcitonin generelated peptide in the epidermis to be reduced in number after anti-NGF treatment (G.R. Lewin, J.R. Tonra, S.B. McMahon, and L.M. Mendell, unpublished observations).

The importance of NGF for cell survival during embryonic and early postnatal periods has been well documented (Barde, 1989). By the time animals have reached adulthood, peripherally derived NGF is no longer important as a survival factor in the intact animal (however, see Johnson and Yip, 1985, for a discussion of centrally derived NGF). In the adult, NGF still regulates some aspects of sensory neuron function, in particular neuropeptide levels (Mayer et al., 1982), indicating that as animals mature the function of NGF changes. Our findings indicate that the ability of anti-NGF treatment to cause cell death ends by PND 2. Beyond PND 2, but within a time frame during which the innervation of skin is still developing, we demonstrate that NGF is capable of influencing the character of skin innervation. Thus, as animals continue to mature, a different role for NGF is revealed, restricted to a short period during development and independent of the effects of NGF on cell survival.

\section{Conclusions}

We have presented evidence to suggest that NGF deprivation can induce a phenotypic switch in developing primary afferents. This switch, from an HTMR (A $\delta$ ) to a low-threshold mechanoreceptor (D-hair), can occur in the absence of anti-NGFinduced cell death and is restricted to a critical period in early postnatal life corresponding to a time when there is an anatom- 


\section{PHYSIOLOGY}

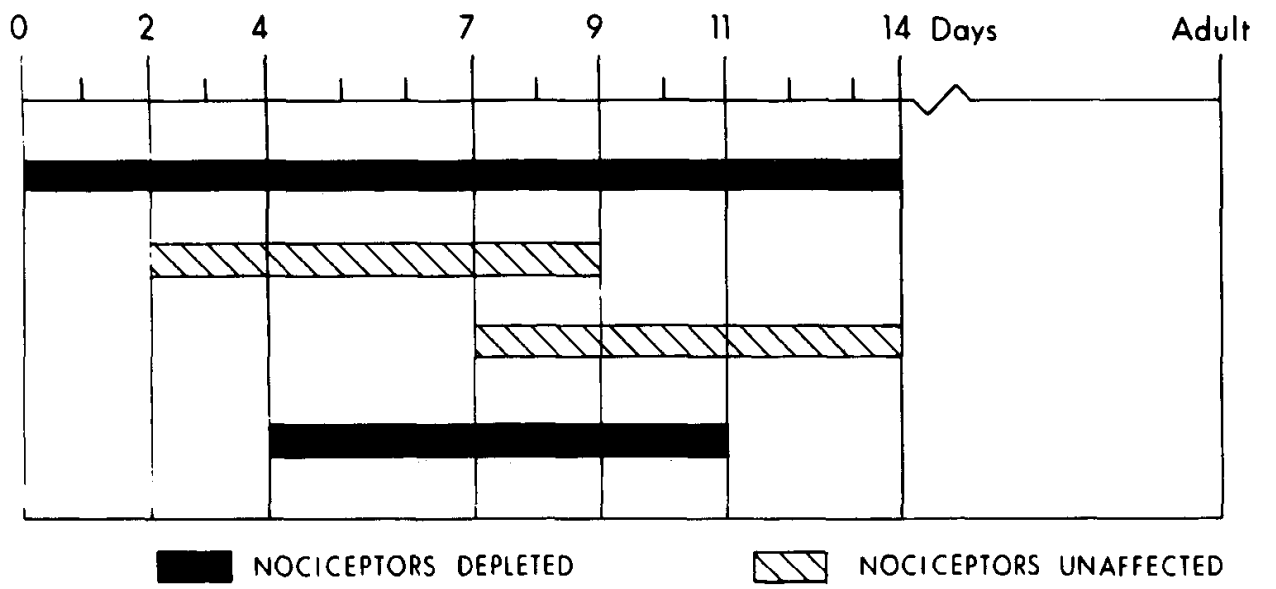

A\& Nociceptors

Depleted

Yes

No

No

Yes

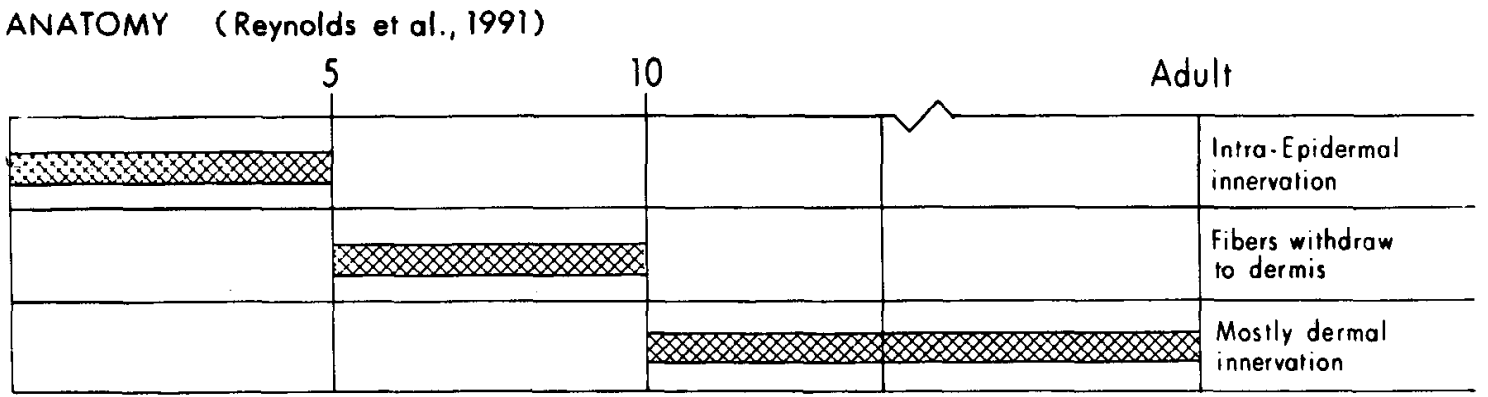

Figure 7. Summary of the timing of effective and ineffective anti-NGF treatments in relation to the timing of anatomical changes in skin innervation. Note that treatments are designated ineffective when there was no statistically significant decrease in the number of HTMRs found in the sural nerve.

ical change in skin innervation. This finding implies that the phenotype of this population of thinly myelinated afferents is not irreversibly specified at birth, and that these afferents require information from the periphery in order to stabilize their identities. One can only speculate about the nature of this information, whether it is a factor derived from the dermis or from the hair follicle, and whether there are perhaps other factors associated with different types of afferents. The fate of the central projections of these converted afferents remains as yet unknown. Further experiments are under way to determine whether their central terminations have arranged themselves to become appropriate anatomically and functionally to their new targets.

\section{References}

Barde Y-A (1989) Trophic factors and neuronal survival. Neuron 2: 1525-1534.

Burgess PR, Perl ER (1967) Myelinated afferent fibres responding specifically to noxious stimulation of the skin. J Physiol (Lond) 190: 541-562.

Davies AM, Bandtlow C, Heumann R, Korsching S, Rohrer H, Thoenen $\mathrm{H}$ (1987) Timing and site of nerve growth factor synthesis in developing skin in relation to innervation and expression of the receptor. Nature 326:353-358.

Fitzgerald M (1987a) Spontaneous and evoked activity of fetal primary afferents in vivo. Nature 326:603-604.

Fitzgerald $M$ (1987b) Cutaneous primary afferent properties in the hindlimb of the neonatal rat. J Physiol (Lond) 383:79-82.

Fitzgerald MJT (1967) Perinatal changes in epidermal innervation in rat and mouse. J Comp Neurol 126:37-42.

Friede RL, Samorajske T (1968) Myelin formation in the sciatic nerve of the rat. J Neuropathol Exp Neurol 27:546-571.
Fulton BP (1987) Postnatal changes in conduction velocity and somal action potential parameters of rat dorsal root ganglion neurons. Neurosci Lett 73:125-130.

Goedert M, Fine A, Hunt SP, Ullrich A (1986) Nerve growth factor mRNA in peripheral and central rat tissues and in the human central nervous system: lesion effects in the rat brain and levels in Alzheimer's disease. Mol Brain Res 1:85-92.

Gorin PK, Johnson EM Jr (1980) Effects of long term nerve growth factor deprivation in the nervous system of the adult rat: an experimental autoimmune approach. Brain Res 198:27-42.

Greene LA (1977a) A quantitative bioassay for nerve growth factor (NGF) activity employing a clonal pheochromocytoma line. Brain Res 133:350-353.

Greene LA (1977b) Quantitative in vitro studies on the nerve growth factor (NGF) requirement of neurons. II. Sensory neurons. Dev Biol 58:106-113.

Harper AA, Lawson SN (1985) Conduction velocity is related to morphological cell type in rat dorsal and root ganglion neurons. J Physiol (Lond) 359:31-46.

Hohn A, Leibrock J, Bailey K, Barde Y-A (1990) Identification and characterization of a novel member of the nerve growth factor/brain derived neurotrophic factor family. Nature 344:339-341.

Horch KW, Tuckett RD, Burgess PR (1977) A key to the classification of cutaneous mechanoreceptors. J Invest Dermatol 69:75-82.

Hulsebosch CE, Perez-Polo JR, Coggeshall RE (1987) In vivo antiNGF induces sprouting of sensory axons in dorsal roots. J Comp Neurol 259:445-451.

Johnson EM Jr, Yip HK (1985) Central nervous system and peripheral nerve growth factor provide trophic support critical to mature sensory neuronal survival. Nature 314:751-752.

Johnson EM Jr, Gorin PD, Brandeis LD, Pearson J (1980) Dorsal root ganglion neurons are destroyed by exposure in utero to maternal antibody to nerve growth factor. Science 219:916-918.

Korsching S, Thoenen H (1983) Nerve growth factor in sympathetic 
ganglia and corresponding target organs of the rat: correlation with density of sympathetic innervation. Proc Natl Acad Sci USA 80: 3513-3516.

Kruger L, Perl ER, Sedivec MJ (1981) Fine structure of myelinated mechanical nociceptor endings in cat hairy skin. J Comp Neurol 198: 137-154.

Landis SC (1990) Target regulation of neurotransmitter phenotype. Trends Neurosci 13:344-350.

Lawson SN (1979) The postnatal development of large light and small dark neurons in mouse dorsal root ganglia: a statistical analysis of cell numbers and size. J Neurocytol 8:275-294.

Lawson SN, Harper AA, Harper EI, Garson JA, Anderton BH (1984) A monoclonal antibody against neurofilament protein specifically labels a subpopulation of rat sensory neurons. J Comp Neurol 228: 263-272.

Lewin GR, McMahon SB (1991) Physiological properties of primary sensory neurons appropriately and inappropriately innervating skin in adult rats. J Neurophysiol 66:1205-1217.

Lewin GR, Ritter AM, Mendell LM (1991a) The dependence of myelinated nociceptors on NGF in the neonatal rat: evidence for a postnatal critical period. J Physiol (Lond) 438:167P.

Lewin GR, Ritter AM, Mendell LM (1991b) Regulation of nociceptor function by NGF in the neonatal and adult rat. Soc Neurosci Abstr 17:546.3.

Lindsay RM, Thoenen H, Barde Y-A (1985) Placode and neural crestderived sensory neurons are responsive at early developmental stages to brain-derived neurotrophic factor. Dev Biol 112:319-328.

Lynn B, Carpenter SE (1982) Primary afferent units from the hairy skin of the rat hind limb. Brain Res 238:29-43.

Lynn B, Shakhanbeh J (1988) Properties of A $\delta$ high threshold mechanoreceptors in the rat hairy and glabrous skin and their response to heat. Neurosci Lett 85:71-76.

Maisonpierre PC, Belluscio L, Squinto S, Ip NY, Furth ME, Lindsay RM, Yancopoulos GD (1990a) Neurotrophin-3: a neurotrophic factor related to NGF and BDNF. Science 247:1446-1451.

Maisonpierre PC, Belluscio L, Friedman B, Alderson RF, Wiegand SJ, Furth ME, Lindsay RM, Yancopoulos GD (1990b) NT-3, BDNF, and NGF in the developing rat nervous system: parallel as well as reciprocal patterns of expression. Neuron 5:501-509.

Mayer N, Lembeck F, Goedert M, Otten U (1982) Effects of antibodies against nerve growth factor in the postnatal development of substance P-containing sensory neurons. Neurosci Lett 29:47-52.

McMahon SB, Lewin GR, Bloom SB (1991) The consequences of long-term topical capsaicin application in the rat. Pain 44:301-310.

Mendell LM, Lewin GR, Ritter AM (1991) The post-natal dependence of myelinated nociceptors on NGF is not mediated by cell death. Soc Neurosci Abstr 17:546.4.

Reynolds ML, Fitzgerald M, Benowitz LI (1991) GAP-43 expression in developing cutaneous and muscle nerves in the rat hindlimb. Neuroscience 41:200-211.

Richardson PM, Verge Issa VMK, Riopelle RJ (1986) Distribution of neuronal receptors for nerve growth factor in the rat. J Neurosci $6: 2312-2321$

Ritter AM (1991) The effects of nerve growth factor and its antisera on physiologically identified sensory neurons. PhD dissertation, State University of New York at Stony Brook.

Ritter AM, Lewin GR, Kremer NE, Mendell LM (1991) Requirement for nerve growth factor in the development of myelinated nociceptors in vivo. Nature 350:500-502.

Rose RK, Rohrlich D (1987) Counting sectioned cells via mathematical reconstruction. J Comp Neurol 263:365-386.

Schwartz JP, Pearson J, Johnson EM Jr (1982) Effect of exposure to anti-NGF on sensory neurons of adult rats and guinea pigs. Brain Res 244:378-381.

Thoenen H, Barde Y-A (1980) The physiology of nerve growth factor. Physiol Rev 60:1284-1335.

Ygge HK, Aldskogius M, Grant G (1981) Asymmetries and symmetries in the number of thoracic dorsal root ganglion cells. J Comp Neurol 202:365-372.

Yip HK, Rich KM, Lampe PA, Johnson EM Jr (1984) The effects of nerve growth factor and its antiserum on the postnatal development and survival after injury of sensory neurons in rat dorsal root ganglia. J Neurosci 4:2986-2992. 